\title{
Acknowledgement to Reviewers of Genealogy in 2019
}

\author{
Genealogy Editorial Office \\ MDPI, St. Alban-Anlage 66, 4052 Basel, Switzerland
}

Published: 7 February 2020

The editorial team greatly appreciates the reviewers who have dedicated their considerable time and expertise to the journal's rigorous editorial process over the past 12 months, regardless of whether the papers are finally published or not. In 2019, a total of 77 papers were published in the journal, with a median time to first decision of 35.4 days and a median time from submission to publication of 59 days. The editors would like to express their sincere gratitude to the following reviewers for their generous contribution in 2019:

\author{
Aarons, Victoria \\ Adeeko, Adeleke \\ Alfano, Barbara \\ Allwork, Larissa \\ Amore, Roy C. \\ Antonopoulou, Vivi \\ Arnaldi, Marta \\ Bayer, Gerd \\ Bergin, Catherine \\ Borry, Pascal \\ Boyd, Brian \\ Boyd, Diane \\ Brown, Adam \\ Bull, Martin \\ Calogero, Stephen \\ Campbell, Bolaji \\ Cannito, Maddalena \\ Carter, Lyn \\ Conversi, Daniele \\ Coombes, Leigh \\ Dahlvik, Julia \\ Dankertsen, Astri \\ Davis, Mary \\ Deepwell, Katy \\ Derby, Melissa \\ Dermott, Esther \\ Docan-Morgan, Sara \\ Dyer, Jenny \\ Eaglestone, Robert \\ Edwards, Rosalind \\ Elgat, Guy \\ Elias, Anwen \\ Evans, Tanya \\ Finch, Helen \\ Fitzpatrick, Esther
}

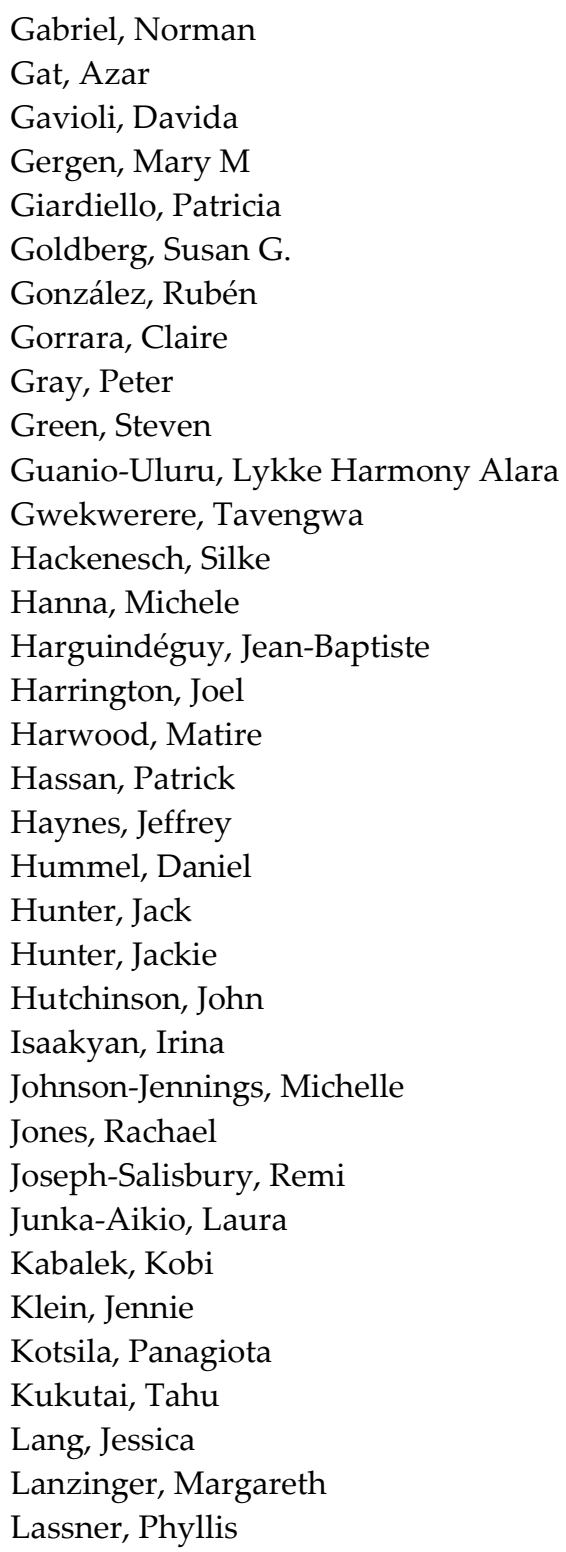

Gabriel, Norman

Gat, Azar

Gavioli, Davida

Gergen, Mary M

Giardiello, Patricia

Goldberg, Susan G.

González, Rubén

Gorrara, Claire

Gray, Peter

Green, Steven

Guanio-Uluru, Lykke Harmony Alara

Gwekwerere, Tavengwa

Hackenesch, Silke

Hanna, Michele

Harguindéguy, Jean-Baptiste

Harrington, Joel

Harwood, Matire

Hassan, Patrick

Haynes, Jeffrey

Hummel, Daniel

Hunter, Jack

Hunter, Jackie

Hutchinson, John

Isaakyan, Irina

Johnson-Jennings, Michelle

Jones, Rachael

Joseph-Salisbury, Remi

Junka-Aikio, Laura

Kabalek, Kobi

Klein, Jennie

Kotsila, Panagiota

Kukutai, Tahu

Lang, Jessica

Lanzinger, Margareth

Lassner, Phyllis 
Leal, Melissa

Liu, Liangni Sally

Lizarazu, Maria Roca

Lundblad-Janjić, Josefina

Smith, Amy M.

Mackay, Finn

Maddox, Raglan

Mahuika, Nepia

Mahuta, Dean

Malisa, Mark

Martín, J.E. Jamal

May, Helen

Medina-Vicent, Maria

Meehan, Catherine

Mengerink, Mark A.

Menke, Martin

Michaud, Francine

Mihailescu, Dana

Mitroiu, Simona

Mroz, Matilda

Munson, Henry

Mutu, Margaret Shirley

Nabizadeh, Golnar

Nackerud, Larry

Newman, Erica

Novoa, Adriana

O'Brien, Valerie M

Ormond. Meghann

Oyewumi, Oyeronke

Paci, Chris

Palaiologou, Ioanna

Perra, Emiliano

Pettitt, Joanne

Pottenger, John R.

Poulios, Ioannis

Prokopiou, Evangelia

Queiroz E Melo, Maria De Fátima
Raleigh, Liz

Ray, Larry

Rodon, Toni

Rogerson, Ann

Roldán, Cristina

Sanjaume-Calvet, Marc

Schumm, Walter R.

Shea, Jennifer M.

Shepherd, John

Shuker, Mary Jane

Silva, Grant J.

Simmonds, Naomi

Slater, Lisa

Smith, Angel

Sørensen, Ninna Nyberg

Staines, Jo

Stevens, Dan

Stewart, Pamela J.

Storie, Monique R.C.

Storm, Eric

Strathern, Andrew J.

Suzack, Cheryl

Tamboli, Vikram

Taylor, James Lance

Teixeira, Carlos

Theobald, Maryanne

Thomson, Stephen

Tyler, Katharine

Van Engeland, Anicee

Van Natta, Meredith

Vargas, Manuel

Vice, Sue

Vincent, Alana

Wong, Wai Ling

Zhang, Zhenjiang

Zou, Yali

(C) 2020 by the author. Licensee MDPI, Basel, Switzerland. This article is an open access article distributed under the terms and conditions of the Creative Commons Attribution (CC BY) license (http://creativecommons.org/licenses/by/4.0/). 